\title{
The effect of long term testosterone replacement therapy on bone mineral density
}

\author{
Fillo $\mathrm{J}^{1}$, Levcikova $\mathrm{M}^{2}$, Luha $\mathrm{J}^{3}$, Ondrus $\mathrm{D}^{4}$ \\ Outpatient Department of Urology, University Hospital Bratislava (Old Town Hospital), \\ Bratislava, Slovakia. dalibor.ondrus@ousa.sk
}

\section{ABSTRACT}

OBJECTIVE: Long-term results of testosterone replacement therapy (TRT) on bone mineral density (BMD) in literature are still missing.

MATERIALS AND METHODS: Totally, 45 males with testosterone deficiency syndrome (TDS) underwent TRT. The mean age was 57.84 years and the follow-up period was 94.62 months. Males were treated with three-month intramuscular injections of $1000 \mathrm{mg}$ testosterone undecanoate. BMD was check at beginning of treatment, after two years and after 5 years. For a statistic evaluation, nonparametric Wilcoxon test was used.

RESULTS: Mean BMD of lumbar spine was 1.067 at beginning, 1.122 after two years and 1.667 and after 5 years. The results after two and also 5 years showed a significant improvement $(p<0.001)$.

CONCLUSION: Authors proved a positive effect of long-term TRT on BMD of the lumbar spine. Densitometry of the whole hip showed also an improvement, but only after 5 years. Densitometry of the femoral neck was relatively stable. Important is that despite the fact that males became older, BMD values of the lumbar spine were improved (Fig. 6, Ref. 18). Text in PDF www.elis.sk.

KEY WORDS: Testosterone deficiency syndrome, testosterone replacement therapy, densitometry, bone mineral density.

\section{Introduction}

There are still many urologists, who doubt a beneficial effect of long term testosterone replacement therapy (TRT) in males with testosterone deficiency syndrome (TDS) and do not offer these males TRT. TRT in males with TDS has multiple positive effect and restores a quality of life (QoL) in affected males. Evaluation of TRT is not so simple because many signs are subjective. One of the most objective sign is densitometry, which measures bone mineral density (BMD). Osteoporosis is an asymptomatic, systemic bone disease characterised by low BMD and microarchitectural deterioration of bone tissue, resulting in an increased bone fragility. Such condition is often underdiagnosed and undertreated, especially in males, therefore considerably increasing the risk of fracture. Of note, fracture-related morbidity and mortality is generally higher in males, partly due to greater frailty. On the other hand, male hypogonadism is defined as the failure of the testes to

${ }^{1}$ Outpatient Department of Urology, University Hospital Bratislava (Old Town Hospital), Bratislava, Slovakia, ${ }^{2}$ Department of Urology, University Hospital Bratislava (St. Cyril \& Method Hospital), Bratislava, Slovakia, ${ }^{3}$ Institute of Medical Biology, Genetics and Clinical Genetics, Comenius University Faculty of Medicine, Bratislava, Slovakia, and ${ }^{4} 1$ st Department of Oncology, Comenius University Faculty of Medicine \& St. Elisabeth Cancer Institute, Bratislava, Slovakia

Address for correspondence: D. Ondruš, MD, DSc, 1st Department of Oncology, Comenius University Faculty of Medicine, St. Elisabeth Cancer Institute, Heydukova 10, SK-812 50 Bratislava, Slovakia. produce androgens, sperm, or both and it is often due to the aging process. This disorder, in turn, causes many systemic disorders, and it is the condition mainly associated with male osteoporosis (1).

\section{Material and methods}

In cross sectional single centre study, totally 45 males with TDS were treated by TRT. The mean age was 57.84 years (range, 42-73) at the time of enrolment. The follow-up period was 94.62 months (range, 19-138; SD 25.997). The males were treated with testosterone undecanoate (TSTU) administered at a dosage of $1,000 \mathrm{mg}$ once every 12 weeks. All of them had the testosterone (TST) level within the physiological range during the follow-up period. The males were regularly checked according to the EAU guidelines (2). BMD was checked at the beginning of treatment, after two years and after 5 years. BMD measurements were made by dual-energy x-ray absorptiometry (DXA) using a Hologic Explorer imaging bone densitometer (QDR Explorer, Hologic, Inc., Bedford, MA, USA) at the lumbal spine (L1-L4) and the femoral neck. Osteoporosis was defined as $\mathrm{T}$ score $\leq 2.5 \mathrm{SD}$, osteopenia as Tscore between -1 and $-2.5 \mathrm{SD}$, and normal BMD as T score $\geq 1 \mathrm{SD}$. For a statistical evaluation, nonparametric Wilcoxon test was used.

\section{Results}

The mean BMD of lumbar spine was 1.067 (range, 0.5661.624; SD 0.209) at the beginning, 1.122 (range, 0.582-1.857; SD 


\section{1-294}

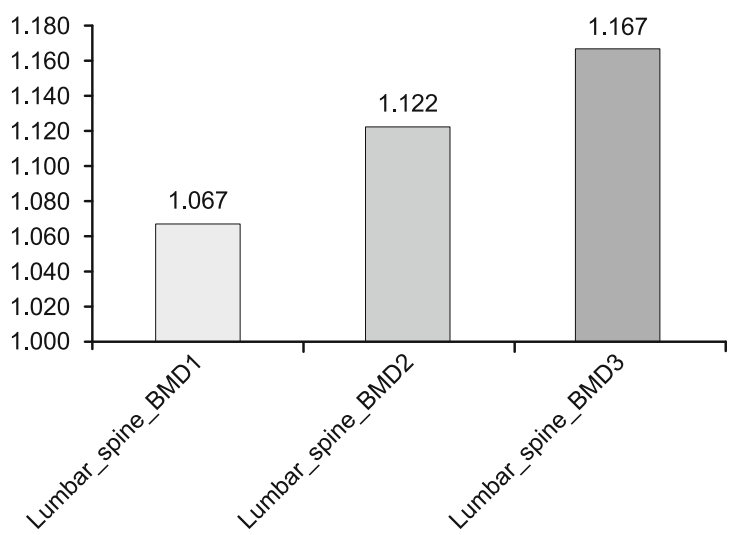

Fig. 1. Lumbar spine BMD at the beginning, after two years, after 5 years.

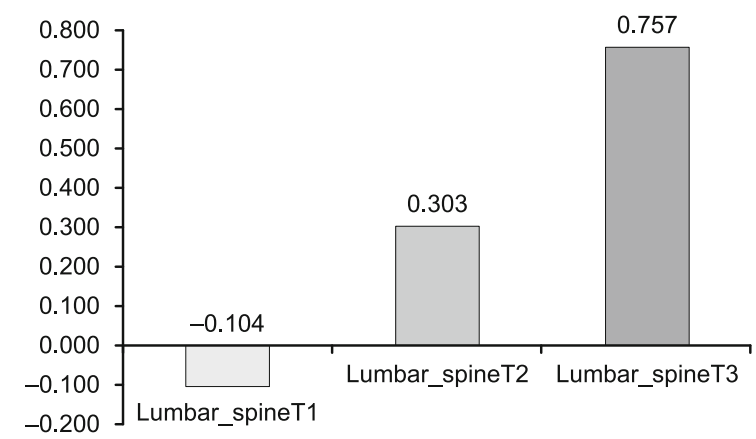

Fig. 2. Lumbar spine $T$ score at the beginning, after two years, after 5 years.

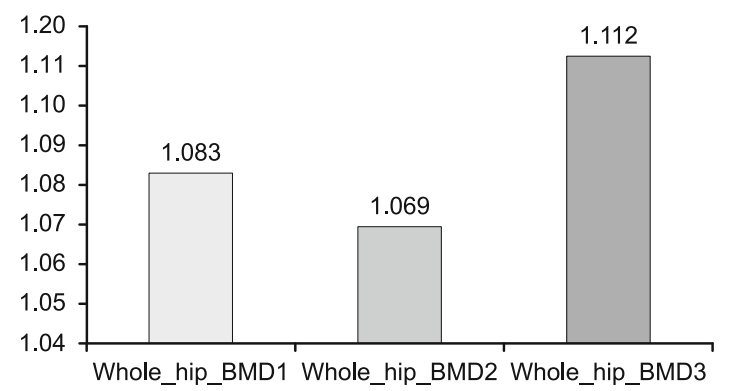

Fig. 3. Whole hip BMD at the beginning, after two years, after 5 years.

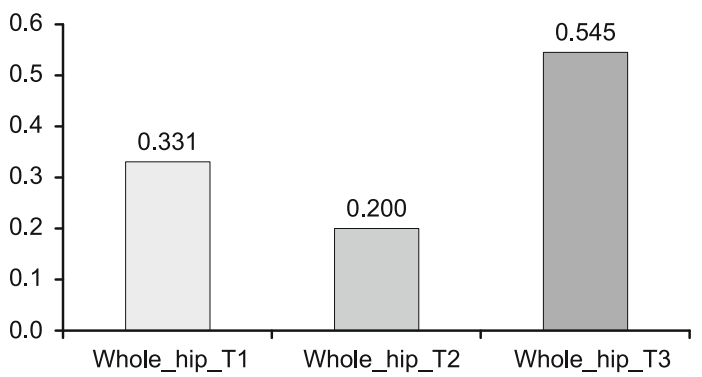

Fig. 4. Whole hip $\mathrm{T}$ score at the beginning, after two years, after 5 years.

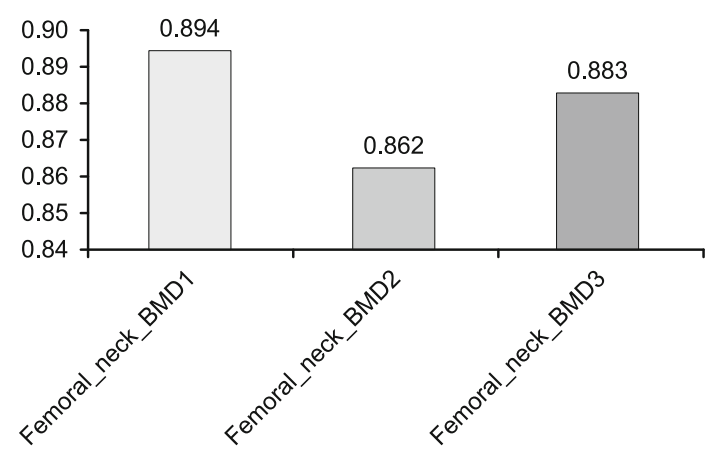

Fig. 5. Femoral neck BMD at the beginning, after two years, after 5 years.

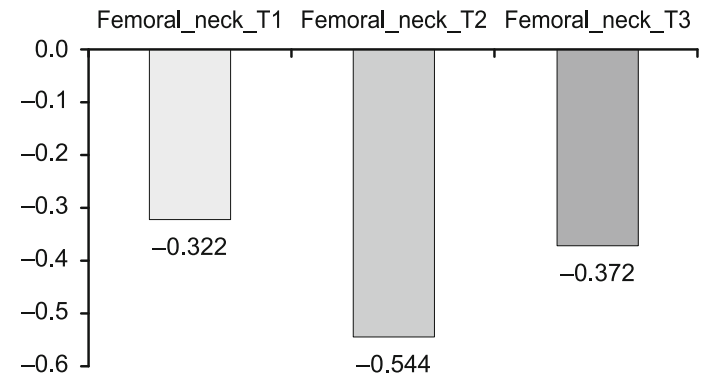

Fig. 6. Femoral neck $T$ score at the beginning, after two years, after 5 years.

0.245 ) after two years and 1.667 (range, 0.913-1.661; SD 0.187) after 5 years. The results after two and after 5 years showed a significant improvement $(\mathrm{p}<0.001)$ (Fig. 1). The mean lumbar spine T score was -0.104 (range, $-3.0-4.7$; SD 1.649) at the beginning, +0.303 (range, -3.0-7.0; SD 1.940) after two years, +0.757 (range, -1.6-4.9; SD 1.685) after 5 years. Improvement was significant $(\mathrm{p}<0.001)$ (Fig. 2). The mean BMD of the whole hip was 1.083 (range, 0.879-1-338; SD 0.103) at the beginning, 1.069 (range, 0.812-1.207; SD 0.097) after two years and 1.112 (range, 0.850-1.388; SD 0.127) after 5 years (Fig. 3). The results showed no improvement after two years, but only after 5 years $(\mathrm{p}<0.001)$ (Fig. 3). The mean $\mathrm{T}$ score of the whole hip was 0.331 (range, -1.0-2.0; SD 0.689) at the beginning, 0.200 (range, -1.5-1.2; SD 0.638) after two years and 0.545 (range, -1.2-2.4; SD 0.876) after 5 years. Significant improvement was observed only after 5 years $(\mathrm{p}<0.001)$ (Fig. 4). The mean BMD of the femoral neck was 0.894 (range, 0.726-1.35; SD 0.121) at the beginning, 0.862 (range, 0.58-1.089; SD 0.103) after two years and 0.883 (range, $0.554-1.128$; SD 0.125) after 5 years, without improvement, but stable results (Fig. 5). The mean $\mathrm{T}$ score of the femoral neck was -0.322 (range, $-1.5-1.2$; SD 0.713) at the beginning -0.544 (range, $-2.6-0.9$; SD 0.763 ) after two years and $-0,372$ (range, -2.80-1.00; SD 0.876) after 5 years (Fig. 6). Au- 
thors did not find any BMD improvement of the femoral neck, but the results were relatively stable despite ageing.

\section{Discussion}

Males aged $>65$ years are subject to an increased incidence of osteoporosis and to increased falls and fractures ultimately contributing to an increased mortality (3). In older males, low serum TST is associated with osteopenia (4) and increased risk of fracture (5). TST administration increases BMD, mainly by suppressing bone resorption $(6,7)$. Males aged $>65$ years with low TST levels are at an increased risk of falls, osteoporosis and fractures $(8-10)$. TRT is usually prescribed to restore optimal TST level, but conflicting data are available about the efficacy of TRT treatment on BMD. The meta-analysis reported a significant increase in lumbar BMD in trials that used intramuscular TST injections, but not in trials that used transdermal TST (11). There are different results of TRT on the lumbar and femoral neck. The controlled trial evaluated the effect of 36 months of TSTU administration on bone health in males with late-onset hypogonadism; this study found substantial increases in both lumbar and femoral neck BMD (12). The randomized, placebo-controlled trial assessing the effects of testosterone enanthate (TSTE) injections on bone health demonstrated improvements in lumbar spine, but not femoral neck BMD (13). Wang et al (14) in their prospective twoyear, single-centre, randomized, placebo-controlled, open-label clinical trial evaluated the efficacy of low-dose TSTU treatment on BMD and biochemical markers of bone turnover in elderly male osteoporosis with low serum TST. Totally, 186 elderly male osteoporosis patients with low serum TST were randomized into three groups: low-dose (LD) TSTU (20 mg, per day), standarddose (40 mg, per day), and placebo group with a 24-month followup. Since the 6th month of the TSTU administration in standarddose group or since the 12th month follow-up in low-dose group and throughout the study, lumbar spine and femoral neck BMD and serum levels of free TST, estradiol, and bone alkaline phosphatase significantly increased. There were no significant differences between the groups of LD TSTU and standard dose TSTU in the percentage of changes of these data since the 18th month of follow-up and throughout the study. No side effects on prostate including PSA were found. LD TSTU (20 mg, per day) may be a cost effective and safe protocol for treating elderly male osteoporosis with low serum TST. With TRT, there was a significant improvement in BMD, both at trabecular and cortical sites. There was a decline in bone turnover with treatment (14). Deb et al (15) proved a positive effect of TRT also in young hypogonadal males. Summary of their study: Short-term parenteral TRT significantly improves BMD at the hip, lumbar spine and forearm in hypogonadal young males.

Another group of younger men with a high level of osteopenia and osteoporosis, who can profit from TRT, are long term survivors of testicular cancer (TC). In the study about TC survivors, Ondrusova et al (16) observed a statistically significantly decreased TST levels (24.35\%) after unilateral orchiectomy (OE) and radiotherapy, in comparison with TC survivors following OE alone or following OE and chemotherapy (14.69\%, $15.48 \%$ respectively). Decrease of TST was observed in $14.74 \%$ of patients with normal BMD and in $20.74 \%$ of patients with an impaired BMD (statistically significant difference). The dependence of BMD on TST values was confirmed.

BMD should be monitored only in males whose BMD was abnormal before the initiation of TRT. An increase in lumbar spine BMD may already be detectable after 6 months of treatment and may continue for three more years (17). Tracz et al (11) in systematic meta-analysis reviewed 658 studies and the results were: intramuscular testosterone moderately increased lumbar BMD in males. The results on femoral neck BMD were inconclusive.

Positive effect of long-term TRT on quality of life was observed also in males with an abdominal obesity (18).

\section{Conclusion}

TRT had multiple positive effects on affected males with TDS and only some suspicious side effects. Evaluation is not so simple because many signs were subjective. The authors used an objective sign densitometry in the current study. The authors proved a positive effect of the long term TRT on BMD in this long-term study in older males, not only on the lumbar spine, but also on the whole hip. Despite the fact that males became older, BMD values were improved. Long term TRT significantly improved the QoL and BMD. Examination of TST levels is an important part of follow-up also in TC survivors (with bilateral as well as unilateral disease). It is necessary to recommend TST examination and subsequent TRT (if needed) to reduce long-term complications including an impaired BMD (osteopenia/ osteoporosis).

\section{References}

1. Tirabassi G, Biagioli A, Balercia G. Bone benefits of testosterone replacement therapy in male hypogonadism. Panminerva Med 2014; 56 (2): $151-163$.

2. Dohle GR, Arver S, Bettocchi C, Jones TH, Kliesch S, Punab M. Guidelines on male hypogonadism 2015; Arnhem (The Netherlands): EAU Central Office; 2015.

3. Bliuc D, Nguyen ND, Alarkawi D, Nguyen TV, Eisman JA, Center JR. Accelerated bone loss and increased post-fracture mortality in elderly women and men. Osteoporosis Int 2015; 26 (4): 1331-1339.

4. Laurent M, Gielen E, Claessens F, Boonen S, Vanderschueren D. Osteoporosis in older men: recent advances in pathophysiology and treatment. Best Pract Res Clin Endocrinol Metab 2013; 27 (4): 527-539.

5. Meier C, Nguyen TV, Handelsman DJ et al. Endogenous sex hormones and incident fracture risk in older men: the Dubbo Osteoporosis Epidemiology Study. Arch Intern Med 2008; 168 (1): 47-54.

6. Beck DT, Yarrow JF, Beggs LA et al. Influence of aromatase inhibition on the bone-protective effects of testosterone. J Bone Miner Res 2014; 29 (11): 2405-2413.

7. Borst SE, Conover CF, Carter CS et al. Anabolic effects of testosterone are preserved during inhibition of 5-reductase. Am J Physiol Endocrinol Metab 2007; 293 (2): E507-E514. 


\section{1-294}

8. Benito M, Gomberg B, Wehrli FW et al. Deterioration of trabecular architecture in hypogonadal men. J Clin Endocrinol Metab 2003; 88 (4): 1497- 1502 .

9. Fink HA, Ewing SK, Ensrud KE et al. Association of testosterone and estradiol deficiency with osteoporosis and rapid bone loss in older men. J Clin Endocrinol Metab 2006; 91 (10): 3908-3915.

10. Greendale GA, Edelstein, S, Barrett-Connor E. Endogenous sex steroids and bone mineral density in older women and men: the Rancho Bernardo Study. J Bone Miner Res 1997; 12 (11): 1833-1843.

11. Tracz MJ, Sideras K, Boloña ER et al. Testosterone use in men and its effects on bone health. A systematic review and meta-analysis of randomized placebo-controlled trials. J Clin Endocrinol Metab 2006; 91 (6): 2011-2016.

12. Aversa A, Bruzziches R, Francomano D et al. Effects of long-acting testosterone undecanoate on bone mineral density in middle-aged men with late-onset hypogonadism and metabolic syndrome: results from a 36 months controlled study. Aging Male 2012; 15 (2): 96-102.

13. Amory JK, Watts NB, Easley KA et al. Exogenous testosterone or testosterone with finasteride increases bone mineral density in older men with low serum testosterone. J Clin Endocrinol Metab 2004; 89 (2): 503-510.
14. Wang YJ, Zhan JK, Huang W et al. Effects of low-dose testosterone undecanoate treatment on bone mineral density and bone turnover markers in elderly male osteoporosis with low serum testosterone. Int J Endocrinol 2013; 2013: 570413 .

15. Deb P Gupta SK, Godbole MM. Effects of short-term testosterone replacement on areal bone mineral density and bone turnover in young hypogonadal males. Indian J Endocrinol Metab 2012; 16 (16): 947-951.

16. Ondrusova M, Spanikova B, Sevcikova K, Ondrus D. Testosterone deficiency and bone metabolism damage in testicular cancer survivors. Am J Men's Health 2018; 12 (3): 628-633.

17. Saad F, Aversa A, Isidori AM, Zafalon L, Zitzmann M, Gooren L. Onset of effects of testosterone treatment and time span until maximum effects are achieved. Eur J Endocrinol 2011; 165 (5): 675-685.

18. Fillo J, Breza J, Ondrusova M et al. Results of long term testosterone replacement therapy in men with abdominal obesity, erectile dysfunction and testosterone deficiency. Bratisl Med J 2018; 119 (8): 577-580.

Received January 9, 2019. Accepted February 11, 2019. 